\title{
Temporal characterization of individual harmonics of an attosecond pulse train by THz streaking
}

\author{
F. Ardana-Lamas, ${ }^{1,2, *}$ C. Erny, ${ }^{1}$ A. G. Stepanov,${ }^{1}$ I. Gorgisyan,,${ }^{1}$ P. Juranić, ${ }^{1}$ R. Abela, ${ }^{1}$ and C. P. Hauri ${ }^{1,2, \dagger}$ \\ ${ }^{1}$ Paul Scherrer Institute, SwissFEL, CH-5232 Villigen PSI, Switzerland \\ ${ }^{2}$ École Polytechnique Fédérale de Lausanne, CH-1015 Lausanne, Switzerland
}

(Received 1 February 2016; published 22 April 2016)

\begin{abstract}
We report on the temporal pulse characteristics of individual harmonics in an attosecond pulse train by means of photoelectron streaking in a strong low-frequency transient. The scheme allows one to retrieve the pulse durations and first-order chirp of individual harmonics without the need for temporal scanning. The measurements were performed using an intense $\mathrm{THz}$ field generated by a tilted phase front technique in $\mathrm{LiNbO}_{3}$. We compared the performance of Xe clusters and atomic $\mathrm{He}$ as a detection medium and retrieved the temporal properties for harmonics of order 19,21, 23, 25, and 27. Our measurements confirm that the individual pulse durations and linear chirp decrease by harmonic order.
\end{abstract}

DOI: null

\section{INTRODUCTION}

High-order-harmonic generation (HHG) [1] is an established technique for the formation of attosecond pulses [2,3] which have been used for recording inner atomic electron dynamics on the subfemtosecond time scale [4]. The HHG process ejects an attosecond burst every half cycle of the driving laser field, which results in the formation of an attosecond pulse train (APT) as a consequence of the coherent summation of individual bursts. In the frequency domain this translates to a regularly separated harmonic comb which eventually goes up to the keV photon energy range [5].

Recently, there has been a rising number of frequencyselective applications which employ only one single individual harmonic of the comb. Examples include free-electron laser seeding [6-8], probing of ultrafast magnetic dynamics at the absorption edges [9-11], as well as time- and angleresolved photoemission spectroscopy (tr-ARPES) [12]. Those dynamics have a corresponding frequency bandwidth similar to the bandwidth of a specific individual harmonic. Thus, for resonant processes, the metrology of an individual harmonic in time becomes essential.

Here, we present a temporal characterization of individual harmonics using the streaking technique [13-16] with a strong terahertz transient. This scheme comes along with the advantage that recording can be performed at a single shot on a time window matching the entire temporal envelope of the entire APT. Presently, different scanning techniques are regularly used for the temporal characterization of APTs, among them reconstruction of attosecond beating by interference of two-photon transitions (RABBIT) [2], frequency-resolved optical gating for complete reconstruction of attosecond bursts (FROG-CRAB) [17], and phase retrieval by omega oscillation filtering (PROOF) [18]. While those scanning techniques provide information about the relative delay between the harmonics (attochirp) and average pulse duration of two neighboring harmonics, information about the chirp and pulse duration of a single harmonic is typically limited to the lower-order harmonics [19], or is still challenging to

\footnotetext{
*fernando.ardana@psi.ch

†christoph.hauri@psi.ch
}

achieve [20]. Cross-correlation techniques with an 800-nm pulse $[21,22]$ as well as the $\mathrm{THz}$ streaking scheme used here overcome those shortages. Compared to the cross-correlation approach, THz streaking is not a scanning technique. While streaking with a few-cycle $800-n m$ pulse has been applied on a subfemtosecond time window for the reconstruction of an isolated attosecond pulse [23], the terahertz streaking scheme is suitable for measuring the temporal shape of the individual harmonics and, in principle, as well as of the entire tens of femtosecond long APT. Terahertz streaking provides a quick characterization of the time structure of an individual harmonic as there is no need for scans. The scheme suits in particular the demands of low-repetition-rate soft x-ray sources including plasma-based x-ray lasers, high-power HHG, and self-amplified spontaneous emission (SASE) free-electron lasers where single-shot temporal characterization is essential.

\section{THz STREAKING}

The measurement of the pulse duration is based on a technique presented for a femtosecond and attosecond streak camera $[23,24]$. The general principle of the streak camera is based on mapping the temporal characteristic of an x-ray beam onto the velocity distribution of an electron beam. In the following calculation, atomic units are used unless specified. The energy distribution of the streaked electrons is given by [23]

$$
S(W)=\left|\int_{-\infty}^{\infty} d t e^{i \phi(t)} \mathbf{d}_{\mathbf{p}(\mathbf{t})} \mathbf{E}_{\mathbf{H}}(t) \exp \left[i\left(W+I_{p}\right) t\right]\right|^{2},
$$

where $d_{p(t)}$ is the dipole transition element, $E_{H}(t)$ the x-ray pulse to be characterized, $W$ the energy of the generated photoelectrons, and $\phi(t)$ the phase accumulated between the generation under the presence of an external streaking field and the detection in the spectrometer. Following Ref. [17] and using the assumptions $U_{p} \ll W_{0}$ and a significantly shorter $\mathrm{x}$-ray pulse than the optical cycle of the streaking field $\left(\tau \ll \frac{1}{\omega_{\mathrm{THz}}}\right)$, the phase term can be approximated to

$$
\phi(t) \approx \sqrt{\frac{8 W U_{p}}{\omega_{\mathrm{THz}}^{2}}} \cos \theta\left(1-\frac{\omega_{\mathrm{THz}}^{2} t^{2}}{2}\right)=\frac{s}{\omega_{\mathrm{THz}}^{2}}\left(1-\frac{\omega_{\mathrm{THz}}^{2} t^{2}}{2}\right),
$$


where $U_{p}=E_{0}^{2} / 4 \omega_{\mathrm{THz}}^{2}$ is the ponderomotive energy of the electron in the streaking $\mathrm{THz}$ field with $E_{\mathrm{THz}}(t)=$ $\tilde{E}_{\mathrm{THz}}(t) \cos \left(\omega_{\mathrm{THz}} t\right)$, with the THz envelope $\tilde{E}_{\mathrm{THz}}(t)$, and the slope of the energy shift, the so-called streaking speed $s=$ $\sqrt{2 W} E_{0}$ [25]. The \pm accounts for the direction of the streaking field towards $(+)$ or away $(-)$ from the electron spectrometer. In the presented experiment the detection is in the plane of the streaking field with a narrow acceptance angle, therefore we assume $\theta=0$. If we assume a Gaussian x-ray pulse,

$$
E_{H}(t)=\exp \left\{\frac{-t^{2}}{4 \tau^{2}}\right\} \exp \left\{-i\left(\omega_{H} t+\xi t^{2}\right)\right\}
$$

with $\tau$ the rms pulse duration and $\xi$ the first-order chirp. The length of the transform-limited pulse $(\widetilde{\tau})$ with the same rms spectral width $\sigma_{0}$ is then given by

$$
\tilde{\tau}^{2}=\frac{\tau^{2}}{1+16 \tau^{4} \xi^{2}}=\frac{1}{4 \sigma_{0}^{2}}
$$

Under these conditions the integral in (1) can now be solved analytically and we obtain

$$
S(W) \propto \exp \left\{\frac{-\left(W-W_{0}\right)^{2}}{2\left(\sigma_{0}^{2}+\tau^{2}\left[s^{2} \pm 4|s| \xi\right]\right)}\right\},
$$

with $W_{0}=\omega_{H}-I_{p}$ the kinetic energy of the unstreaked photoelectrons.

As shown in (5), the broadening produced under the effect of the streaking field depends on the pulse duration as well as on the chirp. By combining the measurements with opposite streaking speeds, e.g., opposite streaking directions, we can calculate directly both the pulse duration and the chirp using the following expressions:

$$
\tau=\sqrt{\frac{\tilde{\sigma}_{+}^{2}+\tilde{\sigma}_{-}^{2}}{2 s^{2}}}, \quad \xi=\frac{\tilde{\sigma}_{+}^{2}-\tilde{\sigma}_{-}^{2}}{8 s \tau^{2}},
$$

where $\tilde{\sigma}_{+/-}=\sqrt{\sigma_{+/-}^{2}-\sigma_{0}^{2}}$ is the deconvoluted rms spectral bandwidth under a positive/negative streaking field. Due to the differential measurement, the technique intrinsically compensates for the systematic spectral broadening as the one produced by the space-charge forces.

To be able to retrieve the pulse duration, a broadening at least equal to the $\mathrm{x}$-ray bandwidth $\left(\frac{s \tau}{\sigma_{0}} \geqslant 1\right.$, for unchirped pulses) needs to be observed [23]. This determines the minimum pulse duration that can be retrieved. To be able to resolve shorter pulses, a higher streaking speed is necessary, however, the approximations made in (2) limit the maximum ponderomotive potential for which an analytical solution exists $\left(U_{p} \ll W_{0}\right)$. In our experiment we estimate a ponderomotive energy of the THz field of $U_{p} \approx 1.2 \mathrm{eV}$, whereas the measured kinetic energies of the photoelectrons are between 8 and $20 \mathrm{eV}$, thus fulfilling the previous condition.

\section{EXPERIMENTAL SETUP}

The experiment was performed using a Ti:sapphire laser system $(20 \mathrm{~mJ}, 100 \mathrm{~Hz})$ with a pulse duration of $39 \mathrm{fs}(\mathrm{rms})$ (Fig. 1). The laser-pulse energy was split to synchronously operate the $\mathrm{THz}$ source $(60 \%)$ as well as the high-orderharmonic generation (40\%).

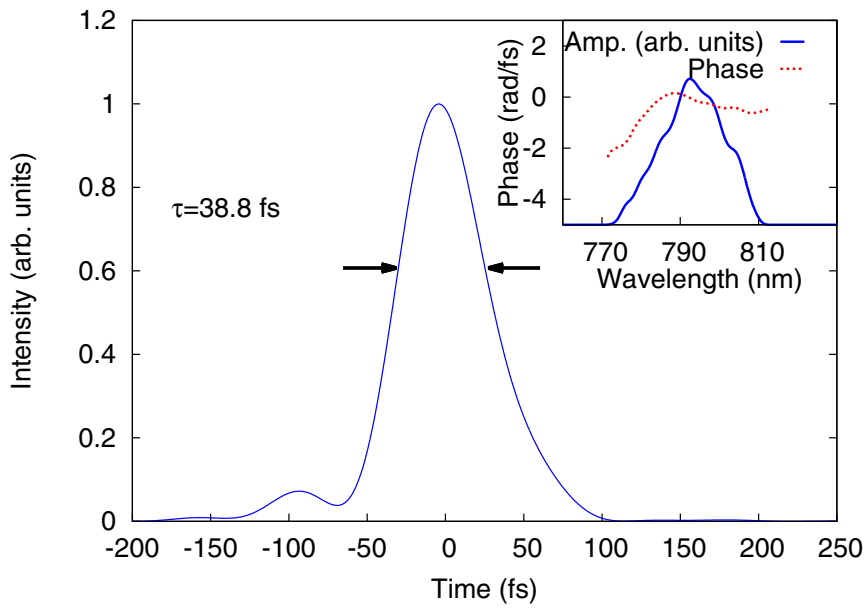

FIG. 1. Temporal profile of the driving laser. The pulse length is 38.8 fs (rms). Inset: Spectral amplitude and phase of the driving pulse, with the latter optimized for most efficient high-order-harmonic generation.

A loose focusing geometry $(f=3 \mathrm{~m})$ is employed for HHG at high flux [26]. The laser waist ( $85 \mu \mathrm{m}, \frac{1}{e^{2}}$ beam radius) was placed at the entrance of a 3-cm-long unmodulated glass cell with an 800- $\mu \mathrm{m}$ inner diameter. A solenoid-based valve situated at the center of the cell released bursts of argon with a backing pressure of up to 3 bars at the full repetition rate of the laser. Downstream the HHG beam is focused by a toroidal mirror (focal length of $4 \mathrm{~m}$ ) into the detection zone of the streaking chamber installed at the end of the 6-m-long HHG beam line. Two 200-nm-thick aluminum filters and a fused silica plate were used to separate the HHG beam from the infrared laser.

$\mathrm{THz}$ radiation is produced directly next to the streaking chamber by optical rectification in a room-temperature $\mathrm{LiNbO}_{3}$ crystal. A tilted pulse front configuration was used to optimize for high-power $\mathrm{THz}$ output [27]. This results in $\sim 10 \mu \mathrm{J}$ pulse energy and a $\mathrm{THz}$ spectrum centered at 0.3 $\mathrm{THz}$ (Fig. 2). The initial vertical $\mathrm{THz}$ polarization is rotated subsequently by a periscope parallel to the HHG polarization.

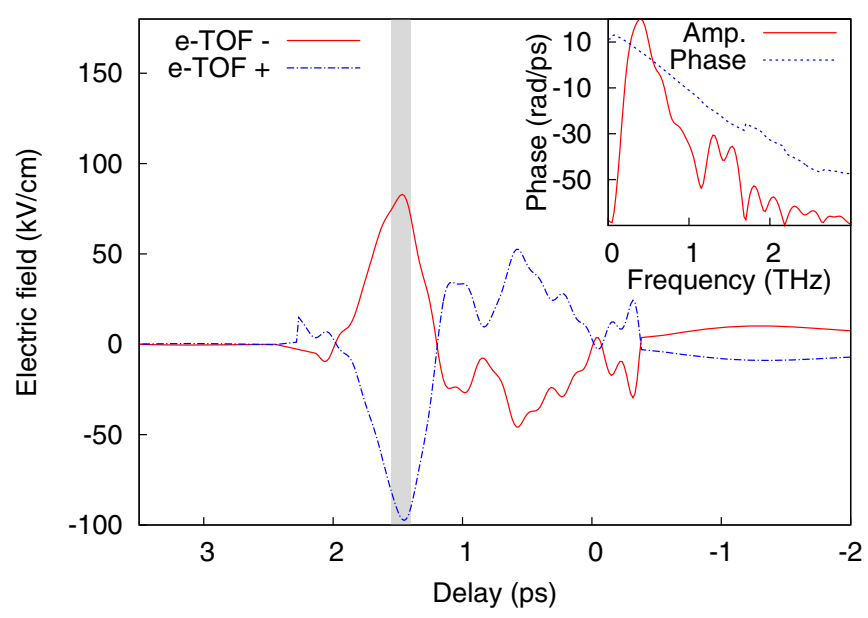

FIG. 2. THz electric field and spectrum obtained by recording photoelectrons produced by the short HHG pulse acting as a probe. 


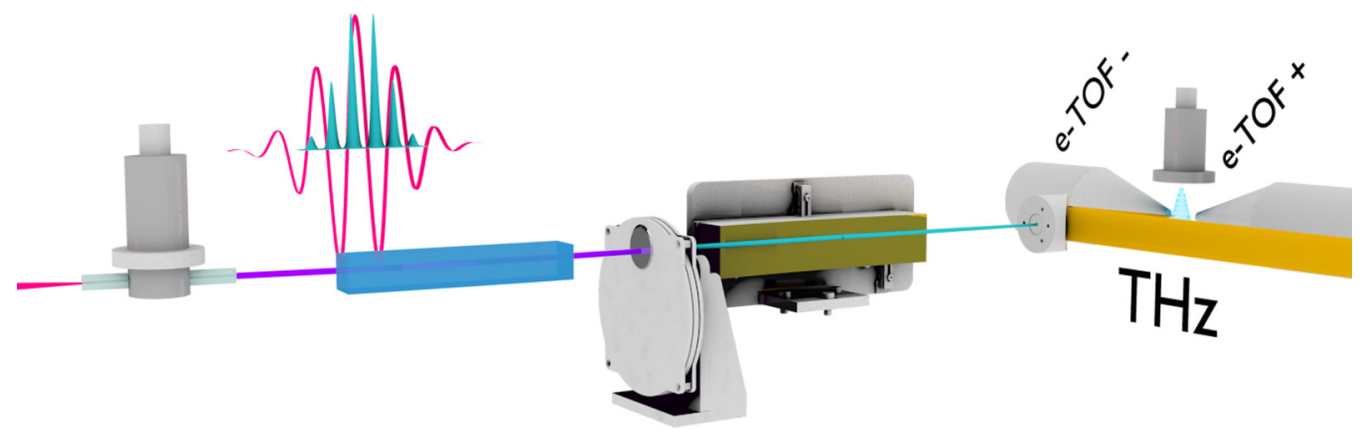

FIG. 3. Experimental layout with high-order harmonics generated in a gas cell followed by harmonic separation (fused silica plate and Al filters) and a 1:1 imaging toroidal mirror (4-m focal length) focusing the APT into the gas jet located in the detection zone of the two opposing time-of-flight electron spectrometers.

After incoupling into the streak camera, the terahertz radiation is overlapped collinearly with the HHG beam using a parabolic mirror $(f=170 \mathrm{~mm})$. For this the latter was equipped with a 5-mm central hole in order to let the HHG beam pass and overlap both beams in time and space. A streaking field strength of up to $100 \mathrm{kV} / \mathrm{cm}$ (Fig. 2) could be achieved at the gas jet position.

The streak camera (Fig. 3) consists of two electron time-offlight (e-TOF) spectrometers oriented parallel to the electric field and placed at opposing sides of the gas jet. For this configuration the recorded signals of the two e-TOFs are complementary as it allows the simultaneous retrieval of positively and negatively streaked electrons (Fig. 2). The typical energy resolution of the e-TOF was around $1 \%$. The photoelectrons were produced by single-photon ionization of either atomic helium or xenon clusters by the HHG beam. Xe builds clusters under the present conditions in the gas jet $[28,29]$. The advantage is the significantly higher absorption cross section of larger and heavier Xe compared to He. This effect is further pronounced due to the cluster formation of $\mathrm{Xe}$. The drawbacks of Xe are the closely spaced photoelectron lines corresponding to the fine-structure thresholds $\mathrm{Xe} 5 p_{3 / 2}$ at $12.1 \mathrm{eV}$ and $\mathrm{Xe} 5 p_{1 / 2}$ at $13.4 \mathrm{eV}$. The gas was injected using a piezocontrolled pulse valve with opening times typically around $20 \mu$ s with a backing pressure between 1 and 4 bars.

\section{EXPERIMENTAL RESULTS}

APT reconstruction requires knowledge about the $\mathrm{THz}$ streaking field. This characterization can be done either by conventional electro-optical sampling or, as we did here, directly by recording the signal of the photoelectrons produced at different delays in the streak field (Fig. 4). Our measurement unveiled a THz field asymmetry between the leading and the trailing edge. This indicates a small chirp of the streaking pulse which could originate from pulse distortion due to absorption in air or from the generation process itself. To avoid inconsistencies we evaluated only the data from the leading edge where the highest streaking speed and highest temporal resolution are achieved. Photoelectron spectra for harmonic 25 from He are shown in Fig. 5 and for harmonic 27 from $\mathrm{Xe}$ in Fig. 6. For both cases the streaked and unstreaked configurations for both e-TOFs are shown. For He a comparison of spectra recorded by the left and right e-TOF, respectively, shows that the corresponding linewidths
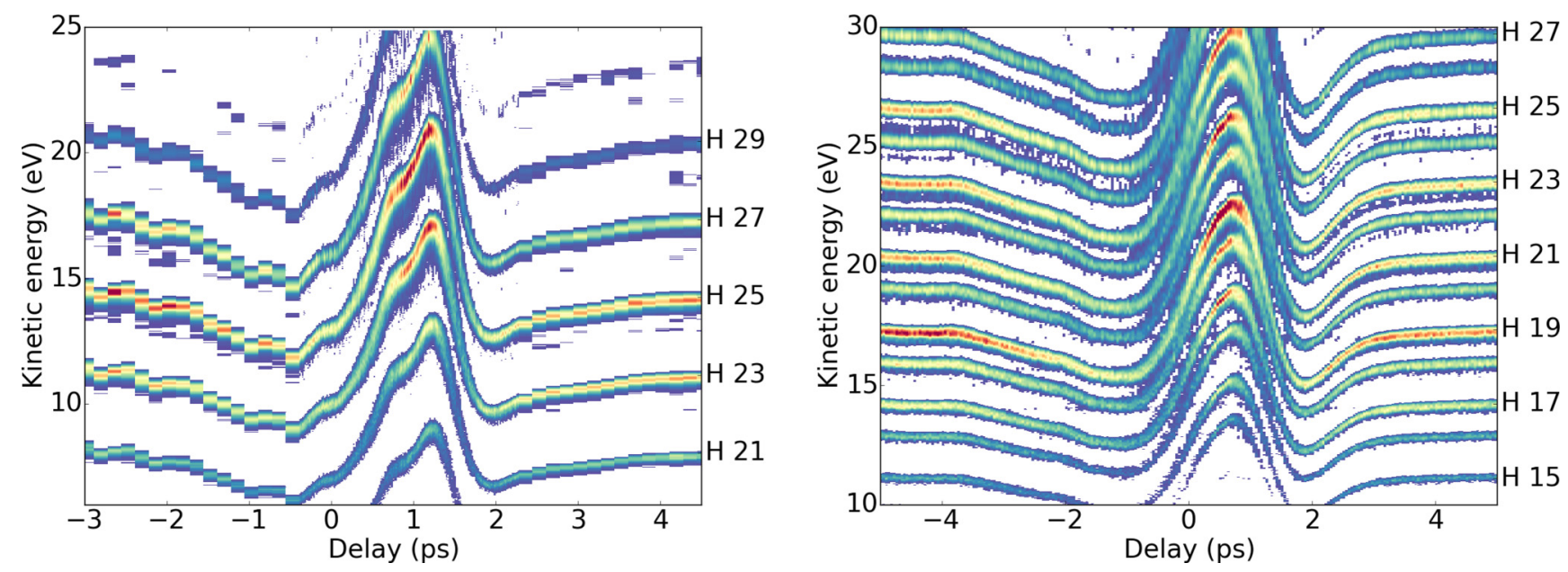

FIG. 4. Photoelectron spectra of individual harmonics generated in He (left) with one ionization line per harmonic order and Xe (right) with ionization lines from Xe $5 p_{3 / 2}$ as well as Xe $5 p_{1 / 2}$ as functions of the time delay between APT and THz field. Higher-order harmonics give rise to higher electron kinetic energies which lead to the observed energy separation of individual harmonics. In order to obtain a better resolution, a finer time step was used around the peak of the THz pulse. 

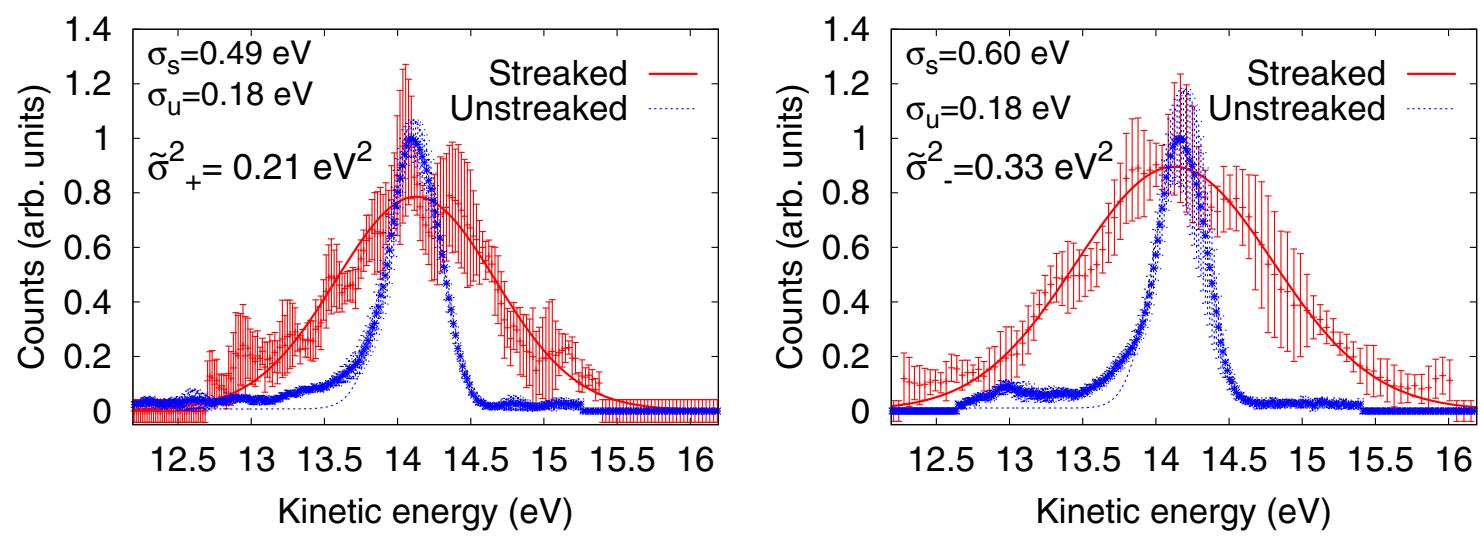

FIG. 5. Left (Right): Comparison of the streaked and unstreaked photoelectron spectra of harmonic 25 from He for the left and right e-TOF. The streaked signals have been shifted in offset in order to overlap with the unstreaked signal.

are differing slightly. According to (6) this entails a linear chirp of about $-1.1 \mathrm{meV} / \mathrm{fs}$ and a pulse duration of $26 \mathrm{fs}$.

While in He a simple single Gaussian assumption was sufficient to fit the central energy as well as the width of the peaks, a double Gaussian with the following form corresponding to the fine-structure components was used for Xe:

$$
\begin{aligned}
I(E)= & A_{a} \exp \left[-\frac{\left(E-E_{a}\right)^{2}}{2 \sigma_{a}^{2}}\right] \\
& +A_{b} \exp \left[-\frac{\left(E-E_{b}\right)^{2}}{2 \sigma_{b}^{2}}\right]+D .
\end{aligned}
$$

For the two Xe lines the fit parameters are given in Table I.

This results in a pulse duration of $27.1 \mathrm{fs}$ for the Xe $5 p_{3 / 2}$ measurement and $32.1 \mathrm{fs}$ for the Xe $5 p_{1 / 2}$ line for harmonic 27. On the other hand, the retrieved chirp values, i.e., -0.56 and $1.61 \mathrm{meV} / \mathrm{fs}$, respectively, vary largely and are neither consistent in value nor in sign. The inconsistency comes from the following issue. Even though the Gaussian fits are still reasonable for the unstreaked spectra, the corresponding peaks are overlapping in the streaked case. Therefore a proper fitting and estimation of the peak width is no longer possible.

For a more accurate retrieval of the pulse duration we have additionally performed a temporal scan around the first maximum of the electric field (corresponding to the linear part of the vector potential) with subsequent Fourier filtering to eliminate discontinuities between subsequent data points and to reduce the noise on the retrieval of $W$ and $\sigma_{+/-}$. To have an estimate on the error on the measurement, five data sets were collected and for each delay the pulse duration and chirp have been calculated. The results were averaged, and the standard deviation used as an estimation of the measurement error. The reference spectra were taken by delaying the THz field by $4 \mathrm{ps}$ compared to the harmonics. At this delay no $\mathrm{THz}$ influence was measurable (Fig. 4). It can be observed (see Fig. 7) how the retrieved pulse duration is only valid in the region where the approximation made in (2) holds (Fig. 7, gray area). In our case we set the limit where the streaking speed deviates by less than $20 \%$ from the maximum. Outside this window the pulse characterization fails and the calculated pulse duration is clearly overestimated. The pulse duration and chirp have been calculated as the weighted average of the data inside of the evaluation window. The minimum pulse duration we are able to retrieve with the current configuration can be calculated by taking into account the linewidth of the unstreaked spectrum and the streaking speed using Eqs. (6) and (4).

In our case, for $\mathrm{He}, \sigma_{0}=0.18 \mathrm{eV}$ and $s=0.02 \mathrm{eV} / \mathrm{fs}$ support the retrieval of a minimum pulse duration of about $10 \mathrm{fs}$ (rms). The pulse length for harmonics of increasing
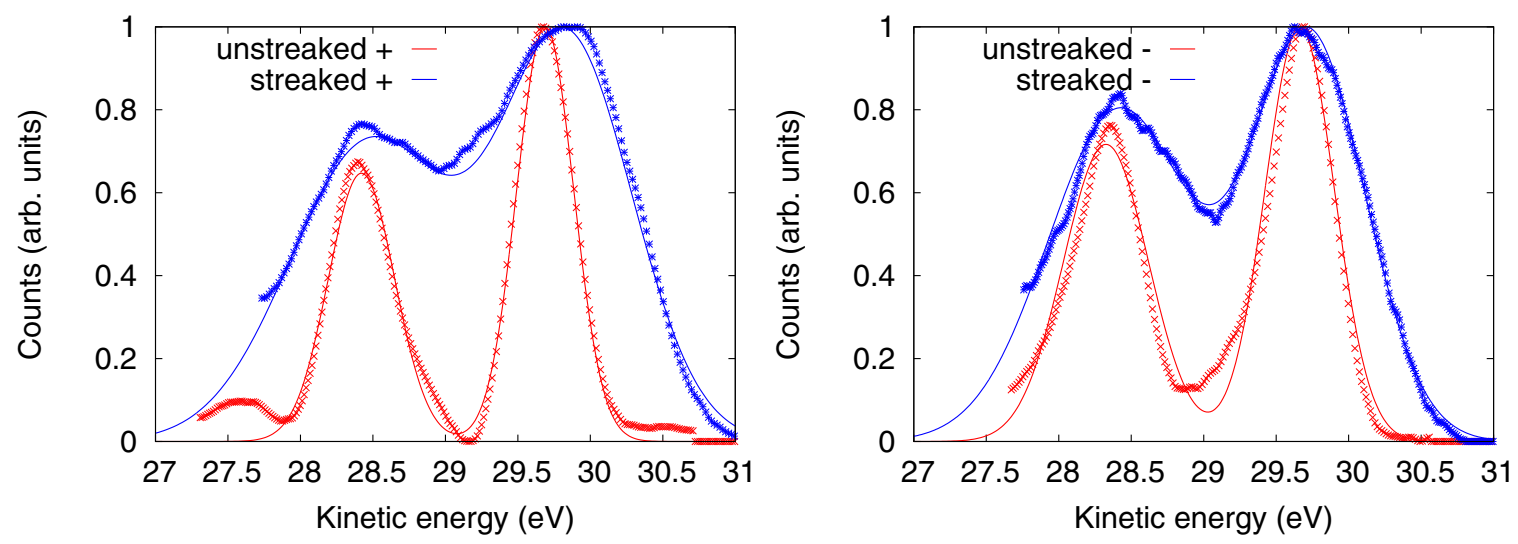

FIG. 6. Left (Right): Comparison of the streaked and unstreaked photoelectron spectra of harmonic 27 from Xe for positive (left) and negative (right) streaking speeds. The streaked signals have been shifted in offset in order to overlap with the unstreaked signal. 
TABLE I. Fitting parameters for harmonic 27 from Xe for (a) $\mathrm{Xe} 5 p_{3 / 2}$ and (b) Xe $5 p_{1 / 2}$.

\begin{tabular}{lcccc}
\hline \hline e-TOF & $\sigma_{a}(\mathrm{eV})$ & $s_{a}(\mathrm{meV} / \mathrm{fs})$ & $\sigma_{b}(\mathrm{eV})$ & $s_{b}(\mathrm{meV} / \mathrm{fs})$ \\
\hline+ & 0.17 & 12.7 & 0.10 & 13.0 \\
- & 0.11 & -14.3 & 0.28 & -14 \\
\hline \hline
\end{tabular}

order $(23,25$, and 27) is decreasing from 36 to 32 and 24 fs (rms), respectively (see Table II), as expected from the highly nonlinear intensity-dependent HHG process. This trend is in agreement with the cutoff law of the HHG process, where the lower-order harmonics can be generated at lower intensities, thus earlier in the leading edge and later in the trailing edge of the pulse, than the higher orders. The measured pulses show a clear negative chirp for the He measurements, which is in agreement with earlier measurements [22,30]. The pulse duration retrieved from Xe shows a significantly larger spread, but taking into account the estimated error, it is still within the range of the values from He. Also, the trend towards shorter pulses with a higher harmonic order is still visible, even though it is less clear. On the other hand, no consistent result can be seen from the chirp measurements. They even contradict the expected negative chirp. As seen in Fig. 6, and mentioned above, the applied $\mathrm{THz}$ field leads to a too strong streaking of the Xe lines, such that the neighboring ionization lines are overlapping, and a reliable estimation of the actual bandwidth is no longer possible. The situation could only be improved by a significantly weaker $\mathrm{THz}$ field, which would come at the cost of lower time resolution, and is there for no suitable path to follow.
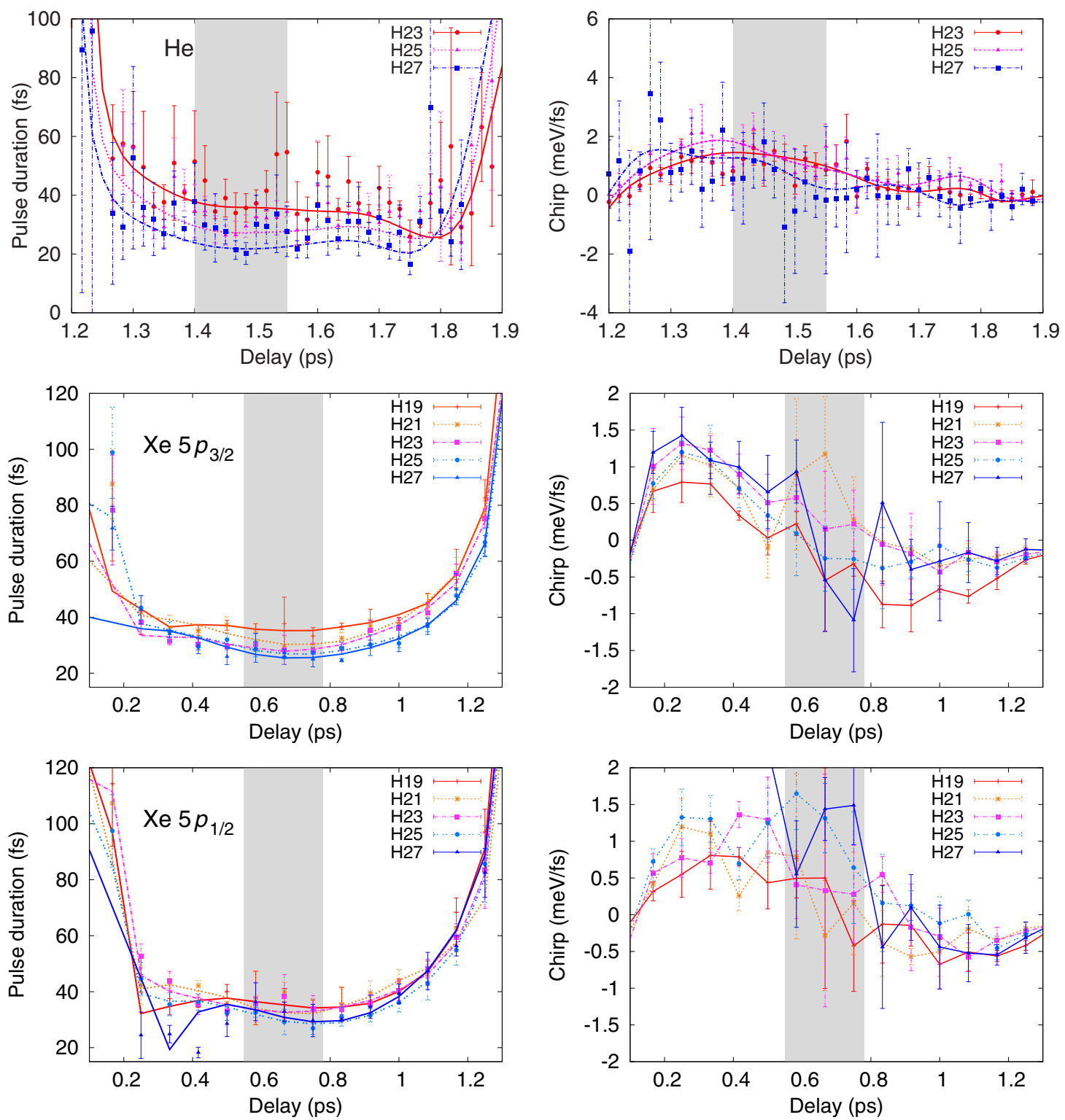

FIG. 7. Retrieved pulse duration (left) and chirp (right) as a function of the time delay for He (top), Xe $5 p_{3 / 2}$ (middle), and Xe $5 p_{1 / 2}$ (bottom). Discrete points: Averaged data over a set of measurements. The solid line represents the smoothed signal using a low-pass frequency filter. The gray area indicates the window where pulse reconstruction according to (2) is valid. Pulse durations and chirp for individual harmonics are summarized in Table II. 
TABLE II. Summarized results from Fig. 7. The pulse duration and chirp are calculated as the weighted average over the region where the approximation $\cos \omega_{\mathrm{THz}} t \approx 1-\frac{\omega_{\mathrm{THz}}^{2} t^{2}}{2}$ is valid (gray area).

\begin{tabular}{lcccc}
\hline \hline Gas/line & $\begin{array}{c}\text { Harmonic } \\
(-)\end{array}$ & $\begin{array}{c}\text { Pulse length } \\
(\mathrm{rms})(\mathrm{fs})\end{array}$ & $\begin{array}{c}\text { Chirp } \\
(\mathrm{meV} / \mathrm{fs})\end{array}$ & $\begin{array}{c}\text { Transform limit } \\
(\mathrm{rms})(\mathrm{fs})\end{array}$ \\
\hline $\mathrm{He}$ & 23 & $36 \pm 5$ & $-0.96 \pm 0.08$ & $4.7 \pm 0.7$ \\
$\mathrm{He}$ & 25 & $32 \pm 4$ & $-0.91 \pm 0.1$ & $6 \pm 1$ \\
$\mathrm{He}$ & 27 & $24 \pm 2$ & $-0.82 \pm 0.1$ & $8 \pm 1$ \\
$\mathrm{Xe} 5 p_{3 / 2}$ & 19 & $36 \pm 11$ & $-0.2 \pm 0.1$ & N/A \\
$\mathrm{Xe} 5 p_{3 / 2}$ & 21 & $30 \pm 6$ & $0.21 \pm 0.05$ & N/A \\
$\mathrm{Xe} 5 p_{3 / 2}$ & 23 & $29 \pm 4$ & $0.5 \pm 0.2$ & N/A \\
$\mathrm{Xe} 5 p_{3 / 2}$ & 25 & $28 \pm 3$ & $-0.28 \pm 0.01$ & N/A \\
$\mathrm{Xe} 5 p_{3 / 2}$ & 27 & $25 \pm 9$ & $-0.6 \pm 0.2$ & N/A \\
$\mathrm{Xe} \mathrm{5} 5 p_{1 / 2}$ & 19 & $35 \pm 5$ & $0.3 \pm 0.2$ & N/A \\
$\mathrm{Xe} 5 p_{1 / 2}$ & 21 & $37 \pm 6$ & $-0.2 \pm 0.1$ & N/A \\
$\mathrm{Xe} 5 p_{1 / 2}$ & 23 & $34 \pm 7$ & $0.5 \pm 0.2$ & N/A \\
$\mathrm{Xe} 5 p_{1 / 2}$ & 25 & $28 \pm 5$ & $-0.9 \pm 0.3$ & N/A \\
$\mathrm{Xe} 5 p_{1 / 2}$ & 27 & $30 \pm 13$ & $1.2 \pm 0.3$ & N/A \\
\hline \hline
\end{tabular}

The full reconstruction of the APT requires further information on the delay between different harmonics, as, e.g., measured by RABBIT. With the presented scheme this is possible with a carrier-envelope-phase (CEP) stable driver laser, or single-shot acquisition with subsequent CEP binning. The CEP drifts lead to timing jitter in the order of the laser period, compared to the $\mathrm{THz}$ field, and is therefore averaged out by this measurement technique. We finally mention that both the streaked and unstreaked photoelectron spectra are broadened by the space-charge forces. However, the pulse reconstruction relies on the difference signal which minimizes these effects.

\section{CONCLUSION}

In summary, we have shown that streaking of photoelectrons by a low-frequency terahertz transient is suitable to measure the pulse duration of the individual harmonics of an attosecond pulse train. We have characterized in $\mathrm{He}$ the individual pulse length and chirp associated with harmonics of order 23-27 generated in a loose-focusing configuration. The linear chirp and pulse durations retrieved from our measurements decrease with harmonic order. With Xe a reconstruction of the pulse duration for harmonics 19-27 was possible, while the chirp could not be retrieved. The transient streaking technique explored here is useful for experiments where the temporal profile of an individual harmonic is required and for single-shot pulse-shape characterization of sources where conventional pulse reconstruction schemes based on scanning are not applicable.

\section{ACKNOWLEDGMENTS}

C.P.H. and F.A. acknowledge financial support from the Swiss National Science Foundation under grant PP00P2 128493, SwissFEL and COST action MP1203 under contract C13.0116.
[1] A. L'Huillier and P. Balcou, Phys. Rev. Lett. 70, 774 (1993).

[2] P. M. Paul, E. S. Toma, P. Breger, G. Mullot, F. Auge, P. Balcou, H. G. Muller, and P. Agostini, Science 292, 1689 (2001).

[3] R. Lopez-Martens, K. Varju, P. Johnsson, J. Mauritsson, Y. Mairesse, P. Salieres, M. B. Gaarde, K. J. Schafer, A. Persson, S. Svanberg, C.-G. Wahlström, and A. L'Huillier, Phys. Rev. Lett. 94, 033001 (2005).

[4] J. Mauritsson, P. Johnsson, E. Mansten, M. Swoboda, T. Ruchon, A. L'Huillier, and K. J. Schafer, Phys. Rev. Lett. 100, 073003 (2008).

[5] T. Popmintchev, M.-C. Chen, D. Popmintchev, P. Arpin, S. Brown, S. Alisauskas, G. Andriukaitis, T. Balciunas, O. D. Mücke, A. Pugzlys, A. Baltuska, B. Shim, S. E. Schrauth, A. Gaeta, C. Hernandez-Garcia, L. Plaja, A. Becker, A. JaronBecker, M. M. Murnane, and H. C. Kapteyn, Science 336, 1287 (2012).

[6] C. Erny, E. Mansten, M. Gisselbrecht, J. Schwenke, R. Rakowski, X. He, M. B. Gaarde, S. Werin, and A. L'Huillier, New J. Phys. 13, 073035 (2011).

[7] G. Lambert, T. Hara, D. Garzella, T. Tanikawa, M. Labat, B. Carre, H. Kitamura, T. Shintake, M. Bougeard, S. Inoue, Y. Tanaka, P. Salieres, H. Merdji, O. Chubar, O. Gobert, K. Tahara, and M.-E. Couprie, Nat. Phys. 4, 296 (2008).
[8] F. Ardana-Lamas, G. Lambert, A. Trisorio, B. Vodungbo, V. Malka, P. Zeitoun, and C. P. Hauri, New J. Phys. 15, 073040 (2013)

[9] G. Lambert, B. Vodungbo, J. Gautier, B. Mahieu, V. Malka, S. Sebban, P. Zeitoun, J. Luning, J. Perron, A. Andreev, S. Stremoukhov, F. Ardana-Lamas, A. Dax, C. P. Hauri, A. Sardinha, and M. Fajardo, Nat. Commun. 6, 6167 (2015).

[10] J. Heidler, C. Piamonteze, R. V. Chopdekar, M. A. UribeLaverde, A. Alberca, M. Buzzi, A. Uldry, B. Delley, C. Bernhard, and F. Nolting, Phys. Rev. B 91, 024406 (2015).

[11] O. Kfir, P. Grychtol, E. Turgut, R. Knut, D. Zusin, D. Popmintchev, T. Popmintchev, H. Nembach, J. M. Shaw, A. Fleischer, H. Kapteyn, M. Murnane, and O. Cohen, Nat. Photonics 9, 99 (2014).

[12] S. Eich, A. Stange, A. V. Carr, J. Urbancic, T. Popmintchev, M. Wiesenmayer, K. Jansen, A. Ruffing, S. Jakobs, T. Rohwer, S. Hellmann, C. Chen, P. Matyba, L. Kipp, K. Rossnagel, M. Bauer, M. M. Murnane, H. C. Kapteyn, S. Mathias, and M. Aeschlimann, J. Electron Spectrosc. Relat. Phenom. 195, 231 (2014)

[13] U. Frühling, M. Wieland, M. Gensch, T. Gebert, B. Schütte, M. Krikunova, R. Kalms, F. Budzyn, O. Grimm, J. Rossbach, E. Plönjes, and M. Drescher, Nat. Photonics 3, 523 (2009).

[14] B. Schütte, U. Frühling, M. Wieland, A. Azima, and M. Drescher, Opt. Express 19, 18833 (2011). 
[15] I. Grguraš, A. R. Maier, C. Behrens, T. Mazza, T. J. Kelly, P. Radcliffe, S. Düsterer, A. K. Kazansky, N. M. Kabachnik, T. Tschentscher, J. T. Costello, M. Meyer, M. C. Hoffmann, H. Schlarb, and A. L. Cavalieri, Nat. Photonics 6, 852 (2012).

[16] P. N. Juranic, A. Stepanov, P. Peier, C. P. Hauri, R. Ischebeck, V. Schlott, M. Radovic, C. Erny, F. Ardana-Lamas, B. Monoszlai, I. Gorgisyan, L. Patthey, and R. Abela, J. Instrum. 9, P03006 (2014).

[17] Y. Mairesse and F. Quere, Phys. Rev. A 71, 011401 (2005).

[18] M. Chini, S. Gilbertson, S. D. Khan, and Z. Chang, Opt. Express 18, 13006 (2010).

[19] Y. Mairesse, O. Gobert, P. Breger, H. Merdji, P. Meynadier, P. Monchicourt, M. Perdrix, P. Salières, and B. Carré, Phys. Rev. Lett. 94, 173903 (2005).

[20] K. T. Kim, D. H. Ko, J. Park, V. Tosa, and C. H. Nam, New J. Phys. 12, 083019 (2010).

[21] J. Norin, J. Mauritsson, A. Johansson, M. K. Raarup, S. Buil, A. Persson, O. Dühr, M. B. Gaarde, K. J. Schafer, U. Keller, C.-G. Wahlström, and A. L'Huillier, Phys. Rev. Lett. 88, 193901 (2002).

[22] J. Mauritsson, P. Johnsson, R. López-Martens, K. Varjú, W. Kornelis, J. Biegert, U. Keller, M. B. Gaarde, K. J. Schafer, and A. L'Huillier, Phys. Rev. A 70, 021801 (2004).
[23] J. Itatani, F. Quere, G. L. Yudin, M. Y. Ivanov, F. Krausz, and P. B. Corkum, Phys. Rev. Lett. 88, 173903 (2002).

[24] E. Constant, V. D. Taranukhin, A. Stolow, and P. B. Corkum, Phys. Rev. A 56, 3870 (1997).

[25] R. Kienberger, E. Goulielmakis, M. Uiberacker, A. Baltuska, V. Yakovlev, F. Bammer, A. Scrinzi, T. Westerwalbesloh, U. Kleineberg, U. Heinzmann, M. Drescher, and F. Krausz, Nature (London) 427, 817 (2004).

[26] X. He, M. Miranda, J. Schwenke, O. Guilbaud, T. Ruchon, C. Heyl, E. Georgadiou, R. Rakowski, A. Persson, M. B. Gaarde, and A. L'Huillier, Phys. Rev. A 79, 063829 (2009).

[27] A. G. Stepanov, L. Bonacina, S. V. Chekalin, and J.-P. Wolf, Opt. Lett. 33, 2497 (2008).

[28] P. N. Juranić, A. Stepanov, R. Ischebeck, V. Schlott, C. Pradervand, L. Patthey, M. Radović, I. Gorgisyan, L. Rivkin, C. P. Hauri, B. Monoszlai, R. Ivanov, P. Peier, J. Liu, T. Togashi, S. Owada, K. Ogawa, T. Katayama, M. Yabashi, and R. Abela, Opt. Express 22, 30004 (2014).

[29] O. F. Hagena and W. Obert, J. Chem. Phys. 56, 1793 (1972).

[30] K. Varjú, Y. Mairesse, B. Carré, M. B. Gaarde, P. Johnsson, S. Kazamias, R. López-Martens, J. Mauritsson, K. J. Schafer, P. Balcou, A. L'huillier, and P. Saliéres, J. Mod. Opt. 52, 379 (2005). 